\title{
5G Uplink Performance of Symbol-Based Schedulers with Network Slicing
}

\author{
Omar Ghanim Al Taee ${ }^{1}$, Ali Othman Al Janaby², Younis M. Abbosh ${ }^{3}$ \\ \{omar.salim2019@stu.uoninevah.edu.iq ${ }^{1}$, ali.mohammed@uoninevah.edu.iq², \\ younis.abbosh@uoninevah.edu.iq ${ }^{3}$ \} \\ Communication Department Ninevah university ${ }^{1,2,3}$
}

\begin{abstract}
One of the key elements of the 5G system is network slicing which is a talented technique to create adapted end-to-end logic network path including dedicated and shared resources. Resources scheduling and distribution of network slices show an essential effect on network performance, resource deployment, and load balancing. This paper compares many resources scheduling schemes in the $5 \mathrm{G}$ system with network slicing. We first compare many resource scheduling algorithms, best CQI (BCQI), Round Robin (RR), proportional fair (PF), to assess each scheme performance. Moreover, this paper proposes an adaptive scheduling scheme that dynamically chooses the scheduling algorithm among mentioned schemes to optimize the traffic, user throughput, and cell capacity. Finally, results anticipated assessed and concluded.
\end{abstract}

Keywords: 5G, Network slicing, Scheduler algorithms.

\section{Introduction}

5G systems are intended to support a wider range of applications and business models [1]. This is probable arises from some very high diversity requirement on network performance to achieve some important parameters; peak rates up to $10 \mathrm{Gbps}$, and latency equal to $1 \mathrm{~ms}$. To optimize the above network parameters for low latency with high user's throughput can achieve peak data rate comes with reduced spectral efficiency (SE). For this situation, utilizing the network slicing technique with a $5 \mathrm{G}$ system has become an initial necessity to enhance $5 \mathrm{G}$ system developers to comprise logical networks with precise functionality, without sacrificing the quality of the infrastructure [2]. This logical network, denoted as slices, can be personalized to achieve two purposes. First to provide a particular system behaviour through the utilization of control plane (CP) or user plane (UP) functions to enhance specific services domain (enhanced MBB (eMBB)), massive Machine Type Communication (mMTC), Ultra-Reliable and Low Latency Communication (URLLC). Second, to offer tenants a specified level of guaranteed network shared resources and isolation concerning the operation of other concurrent slices [2].

Service-defined networking (SDN) and network function virtualization (NFV) are two fundamental technologies for core network slicing [3]. SDN decouples network control and data forwarding, allowing network control operations to run independently in logically centralised controllers as applications [4]. Specific network functions are decoupled from dedicated and hardware platforms and transferred to general-purpose commercial hardware via NFV [5]. Furthermore, as a significant emerging technology in 5G, Mobile Edge Computing 
(MEC) is projected to serve low-latency communication, which is one of the use cases in future $5 \mathrm{G}[6]$.

This paper will deal with the comparison of scheduling schemes used with network slicing. Also, the paper proposed a new scheme that adapts dynamically between different traffic services.

The rest of the paper covers the following: Section 2 debates the previous works, while section 3 overview the types for schedulers. Section 4 presents System Model and Configuration. Then, section 5 discusses Simulation Evaluation and Results Assessment. Section 6 presents the Proposed dynamic scheme. Finally, a conclusion is added at the end of the paper.

\section{Related Works}

Many resources allocation and scheduling schemes have been developed for LTE, LTE-A systems, and finally for $5 \mathrm{G}$ systems, at the uplink, by much research related to performance comparison in terms of packet delay, throughput and, fairness. Some articles as well, investigate the network performance at high-speed UE.

Authors in [7] investigated and measured the downlink performance of the RR, BCQI, and PF schemes in terms of throughput and Bit Error Rate (BER) representing the extreme cases in scheduling. The evaluation indicated that the BCQI scheme beats the other algorithms in terms of user throughput but on the expenditure of fairness to other UEs suffering from weak SNRs. In [8], the authors evaluated the effects of high speed (UEs) on the throughput of different data rate services using four scheduling schemes in the uplink of the LTE system. The paper simulated 20 and 30 UEs switch at high mobility and different service demands.

The authors reported in [9] that the RR and PF schemes performed well in the downlink transmission mode. PF, on the other hand, gave good data rates for many transmission modes. Although RR gave superior compiled and non-BS individual data speeds, the absolute value of this data speed is not as great as the PF.

In [10] research has been carried out stating that, real-time and non-real-time UEs are scheduled individually. More, some UEs perhaps left without getting any resource blocks (RB)s.

Authors in [11] discussed the occasion that has arisen to deepen information in this field of study. This technology is included in phases of standardization and definitions, with every part of which each manufacturer the research group will undertake to refine by offering the most proper solution. One of these aspects is scheduling. The objective of the project is the 5G technology studio as its general construction is exposed for the longest time concerning LTE in cover one and cover two. Then there is concentration on the MAC undercoat to study different algorithm options scheduling that can be applied in the new telephone generation.

The authors of [12] propose a collaborative multi-user pre-emptive scheduling scheme technique to improve system spectral efficiency while lowering latency. Existing URLLC traffic was always given higher precedence during each scheduling opportunity. The supplied scheduler discovers acceptable URLLC-eMBB traffic in a multi-user transmission when irregular URLLC traffic appears during a transmission time interval (TTI). If the available spatial degrees of liberty within a TTI are limited, the URLLC traffic overwrites a portion of the ongoing eMBB transmissions to meet the URLLC latency requirements with little eMBB throughput loss.

In addition, the authors established several scheduling disciplines for public safety users who are required to intervene during exceptional events or in post-disaster areas in [13]. They 
studied which of numerous scheduling methods, such as RR, PF, and others, performed better in this exceedingly difficult setting.

\section{Schedulers Overview}

This paper will compare the performance and delay of many types of scheduling schemes. This item presents a short review of resource allocation and packet scheduling schemes which are: RR, BCQI, and PF.

\subsection{Round Robin (RR) Algorithm}

RR scheme is a non-aware scheduling scheme that lets user equipment (UE) take tries in utilizing the common RBs, without considering the channel states while offering high fairness for all UEs in radio channel assignment. This will degrade the UE throughput [14].

\subsection{Best CQI Algorithm}

This system gives the UE with the highest SNR additional RBs. The channel condition is sent to the BS by the UEs to execute packet scheduling. The BS basically sends the UEs a reference signal (RS) in the downlink. These RSs are used by UEs for channel quality indicator measures (CQI). At the expense of fairness, the BCQI method can boost UE throughput and system capacity. UEs at the cell boundary are unlikely to be scheduled under this technique [15].

\subsection{Proportional Fair (PF) Algorithm}

PF is the scheme used with Time-frequency that was applied between multi-user system. Initially, it utilized Time Domain Scheduling (TDS) system and then Frequency Domain Scheduling (FDS) system. The main purpose of the combined FDS and TDS system is to improve the throughput and data-rate fairness among the UEs diversity [16]. The fairness and throughput are considered for a system model to operate between the BCQI and the RR schemes. The granularity of 1 resource block (RB is proposed to the scheduling algorithm [7].

\section{System Model and Configuration}

gNB controls the scheduling planning for uplink resources between the users. Considering the following gNB and UEs operations the UL transmissions could be shown in Table 1.

Table 1. Operation of gNB and UEs.

\begin{tabular}{|l|l|}
\hline \multicolumn{1}{|c|}{ gNB } & \multicolumn{1}{c|}{ UEs } \\
\hline -Run the scheduling algorithm to assign uplink resour & -Send the pending buffer status report to the gNB. \\
-Send the uplink assignment to the UEs & -Receive the uplink assignment from the UEs. \\
-Receive the PUSCH transmissions from the UEs & $\begin{array}{l}\text {-Adhere to the received uplink assignment from the } \\
\text { gNB for PUSCH transmission. }\end{array}$ \\
\hline
\end{tabular}


Simulation studies are carried out using MATLAB. The simulation model data are integrated in Table 2.

Table 2. CONSTRAINTS DATA IN THE SIMULATIONS.

\begin{tabular}{|l|l|}
\hline Parameters & Settings \\
\hline Configurable & TDD pattern \\
\hline UEs No. & 3 \\
\hline UEs from gNB distances & $\begin{array}{l}\text { UE1:100m, UE2:400m } \\
\text { UE3:700m }\end{array}$ \\
\hline Simulation time & $10000 \mathrm{~ms}$ \\
\hline Scheduling & symbol-based \\
\hline Scheduling strategy comparison between : & RR, BestCQI, PF \\
\hline HARQ & 16 \\
\hline Subcarrier spacing & $15 \mathrm{kHz}$ \\
\hline Duration of the uplink pattern & $5 \mathrm{~ms}$ \\
\hline Periodicity at which the UL packets are generated by UEs & 30 \\
\hline Size of the UL packets (in bytes) generated by UEs & 2000 \\
\hline Sequence number field length (in bits) of the logical channel for each UE & 6 \\
\hline Reassembly timer (in ms) of the logical channel for each UE & 5 \\
\hline The length of Max buffer for logical channel for each UE & 20000 \\
\hline Channel Update Periodicity & $200 \mathrm{~ms}$ \\
\hline
\end{tabular}

\section{Simulation Evaluation and Results Assessment}

The simulation runs using the MATLAB codes is applied to 5G system model. It runs according to the system model parameters model. The MAC and RLC data results for 3 UEs are illustrated in Fig. 1 for the RR Scheduler Note in this algorithm that the distribution is fair according to the user's distance and priority. 

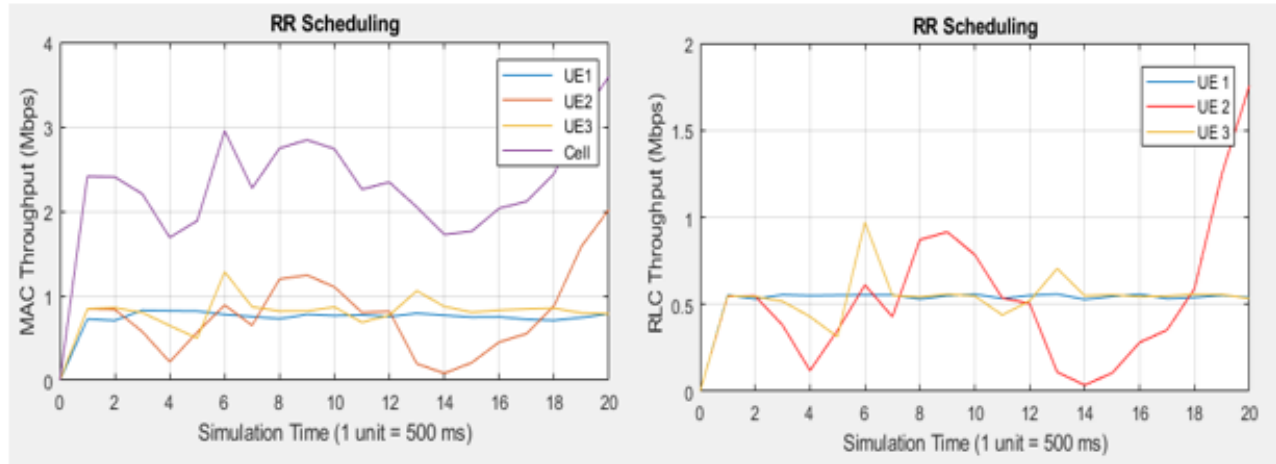

Fig 1. The evaluation user's MAC and RLC throughput with time (RR Scheduler).

According to the results of the user's throughput in Fig 1, it is found that the user's throughput is equal to or slightly more than the cell throughput. And the RLC throughput shown in the figure we found the nearest user is stable and constant with interval.

For the Best CQI scheduler MAC and RLC throughput shown in Fig 2 For this algorithm, the distribution is unfair, and the priority is channel quality.
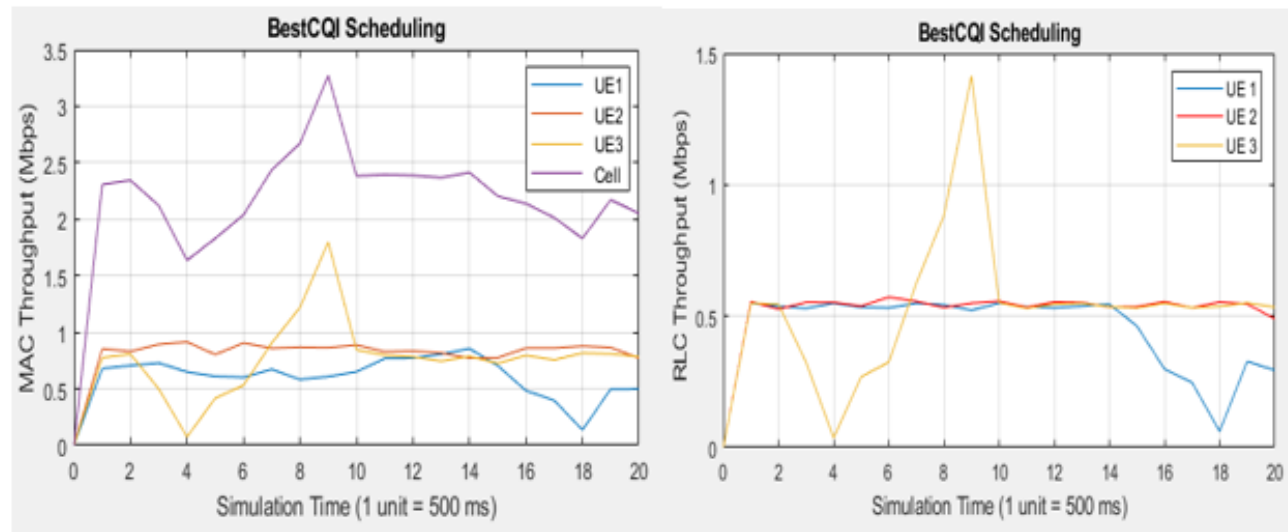

Fig. 2. The evaluation user's MAC and RLC throughput with time (Best CQI Scheduler).

Thus, the results Fig 2 we notice that the user who has higher throughput is the one who has the priority and stability with the interval. When the system uses the PF scheduler the result for MAC and RLC throughput shown in Fig. 3 is a good balance between the objective of maximizing cell throughput and fairness for all users. 

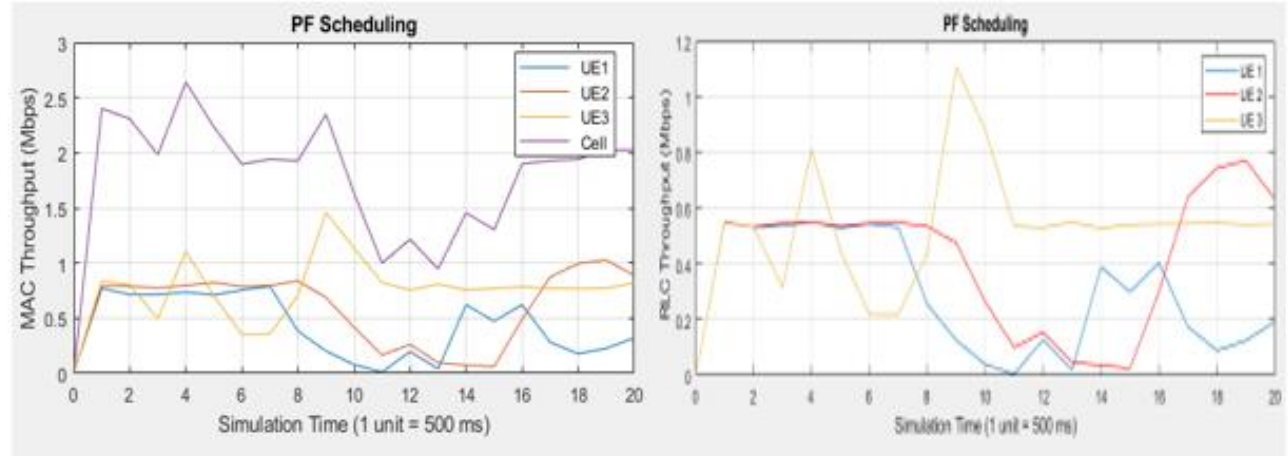

Fig. 3. The evaluation user's MAC and RLC throughput with time (PF Scheduler).

According to Fig 3, we notice balanced distribution among the users because the PF scheduler doesn't rely on interval and priority.

Through the previous results that you get, comparison of types of schedulers we notice that $\mathrm{RR}$ the priority is for the closest Best CQI The priority is for those who have higher CQI, PF all UE has the same opportunity.

To know the distribution of resources, share over time for the 3 type Scheduler, we observe Fig 4,5 and 6.

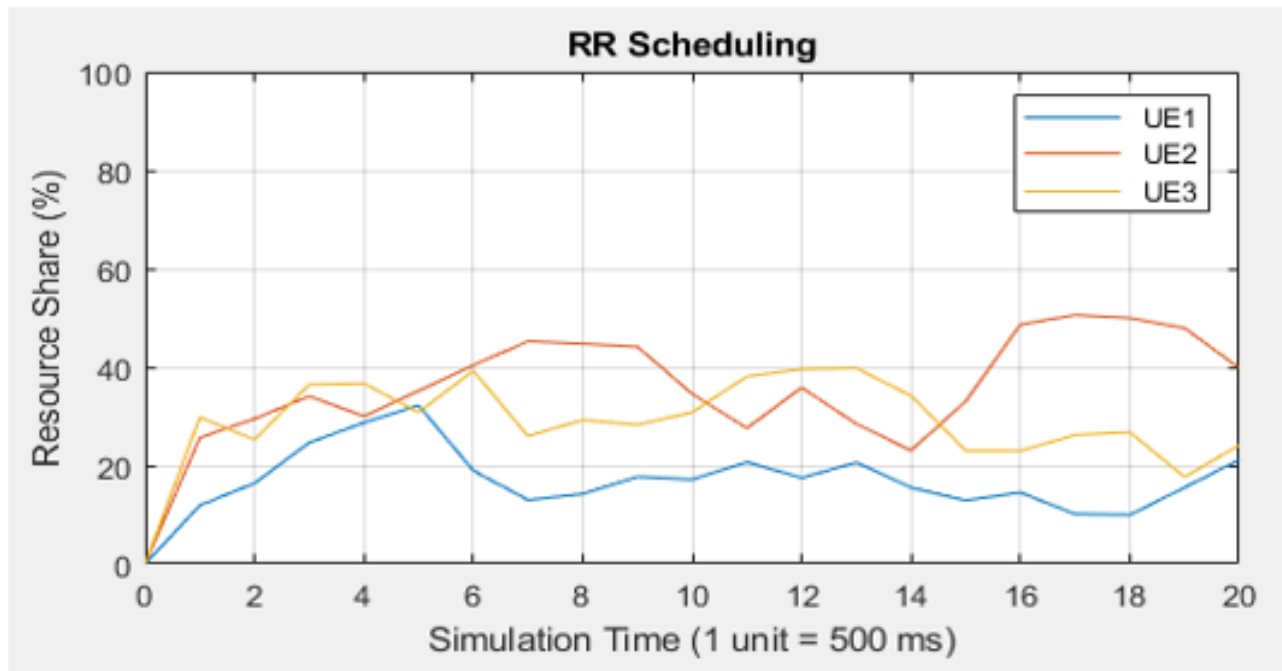

Fig. 4. Resources share with time for RR scheduling. 


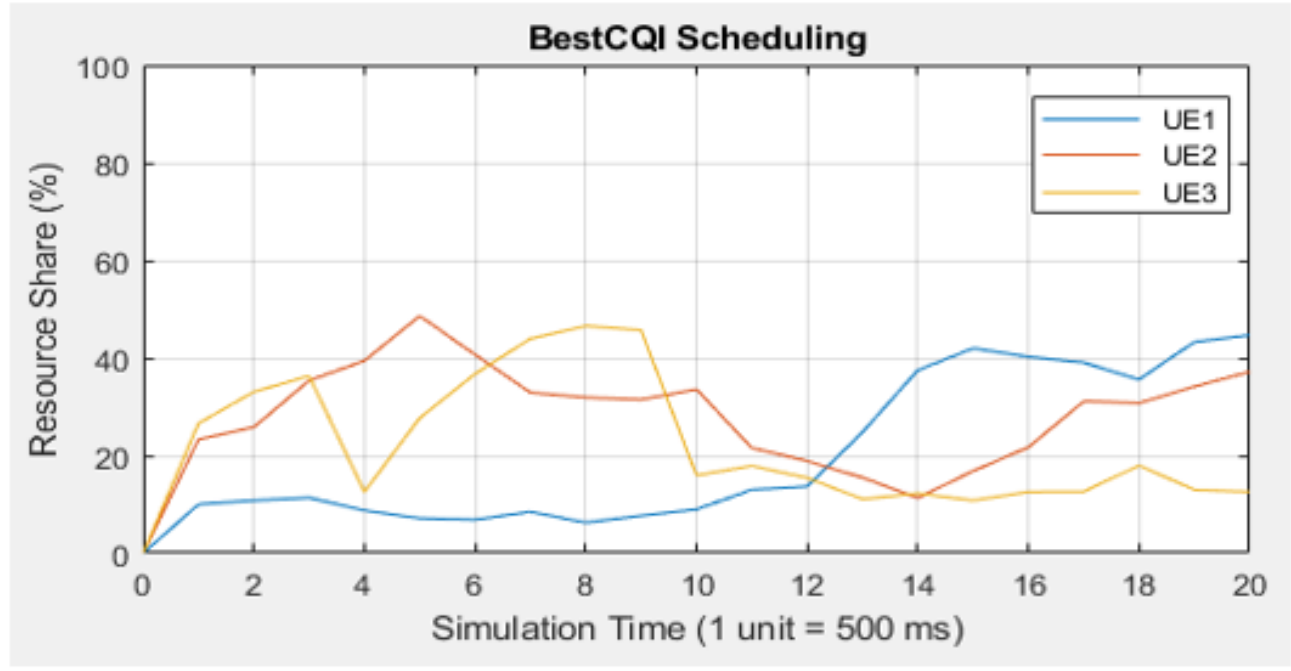

Fig. 5. Resources share with time for BCQI scheduling.

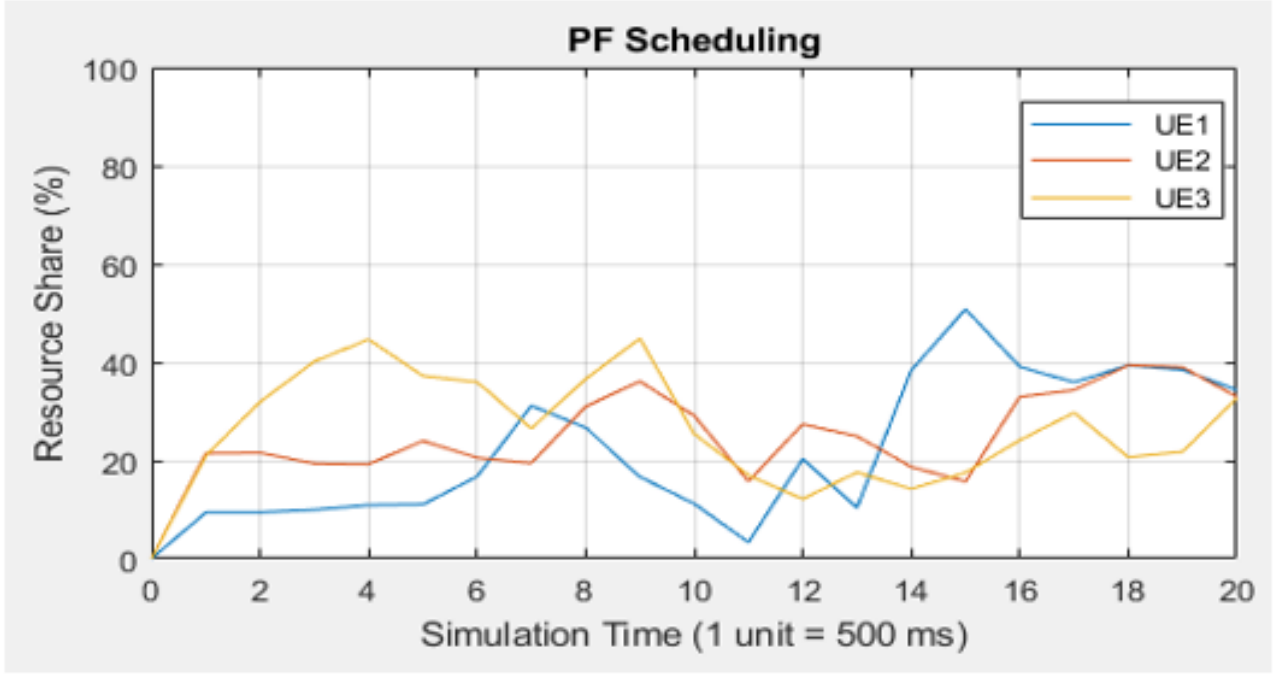

Fig. 6. Resources share with time for PF scheduling.

\section{New Dynamic Proposed Scheme}

According to the channel state for each user, adaptive scheduling can be made according to the users' conditions, for example, if the distance is close between them and the bit you saw is close, RR scheduling can be used, but if there is a miss with CQI and there is nothing emergency, best CQI scheduling can be used, and if there are lapses between users based on terms of capacity and reliability and need a fair procedure we use PF scheduling. 
A scheduler is considered efficient according to the quality of service among UEs. The allocated RBs using BCQI time frame is assigned to the UE with the high CQI (best channel conditions). The BCQI algorithm can approve the system performance. In this scheduling, the eNB controls the located UEs based on the probability to grant resources. It is evidence that BCQI and RR, as well as all scheduling algorithms, could not provide the basis at the same time.

The proposed UL 5G system scheduler is based on this aspect to find a way to achieve the best performances. It leads to a new proposed dynamic UL 5G scheduler. This proposed scheme can switch among BCQI, PF, and RR schedulers since it adaptively add features of all time frames of the operated SNR, the appropriate scheduler for each slice selected according to 5G characteristics shown in Fig 7.
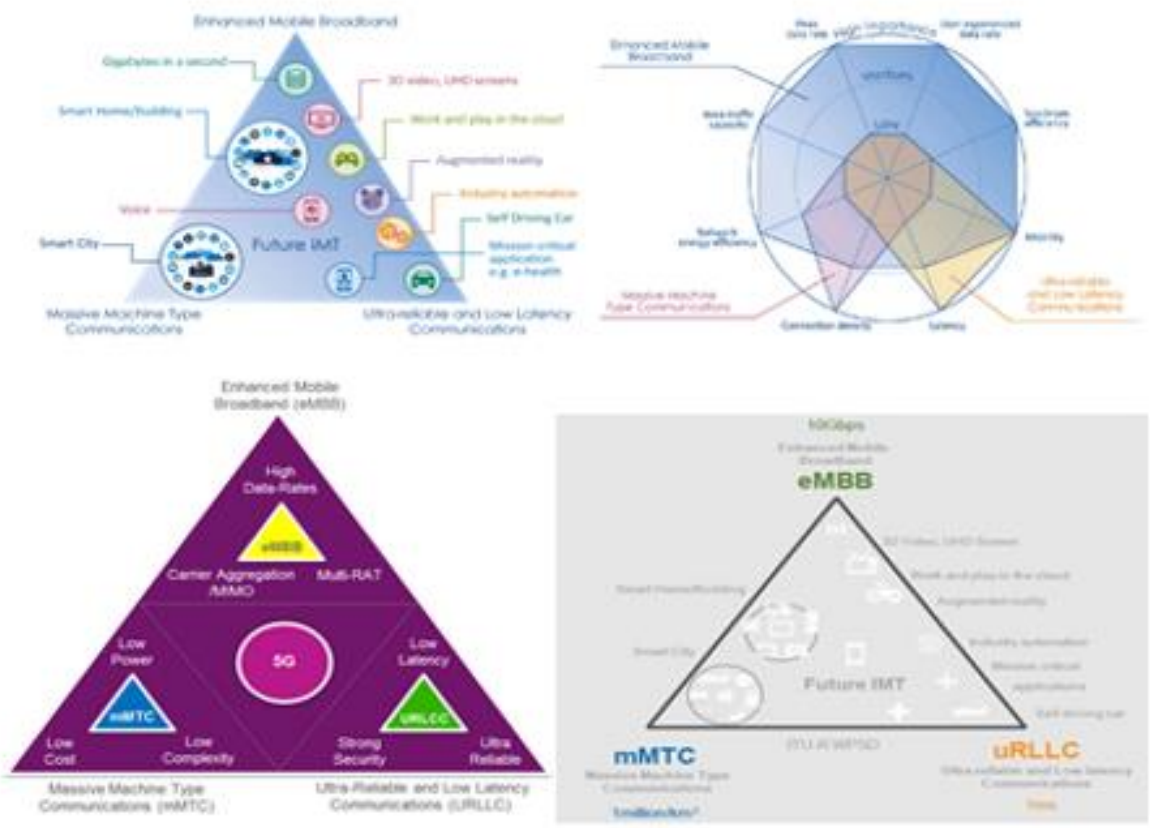

Fig 7. 5G key and Usage Scenarios (eMBB, mMTC, uRLLC).

\subsection{The Dynamic Proposed Scheme Steps}

The proposed adaptive scheme, as indicated in Fig. 8, can be presented as the following:

At each the SINR at the receiver for TTI time slot of all active UEs might be determined. The proposed adaptive scheme inserts a new process; the process is that to investigates whether the user application or service is a traffic type.

The investigation is to choose among traffic types; eMBB, and mMTC. Then, the system assigns the traffic type, a scheduler is adopted according to the following steps.

Step 1: Insert UE's packet into the system's queue. 
Step 2: For each UE, the system finds out CQI, PMI, and RI while taking into consideration the traffic type, channel condition (SNR), and the quality of service (QoS) requirement.

Step 3: The preparation starts at each TTI.

Step 4: System chooses traffic model

Step 5: Each UE assigns his slice

Step 6: For eMBB slice using Best CQI scheduling for URLLC using PF scheduling for mMTC using RR scheduling.

Step 7: System waiting for packet acknowledgment, ACK.

Step 8: if ACK failed, use a hybrid automatic repeat request (HARQ).

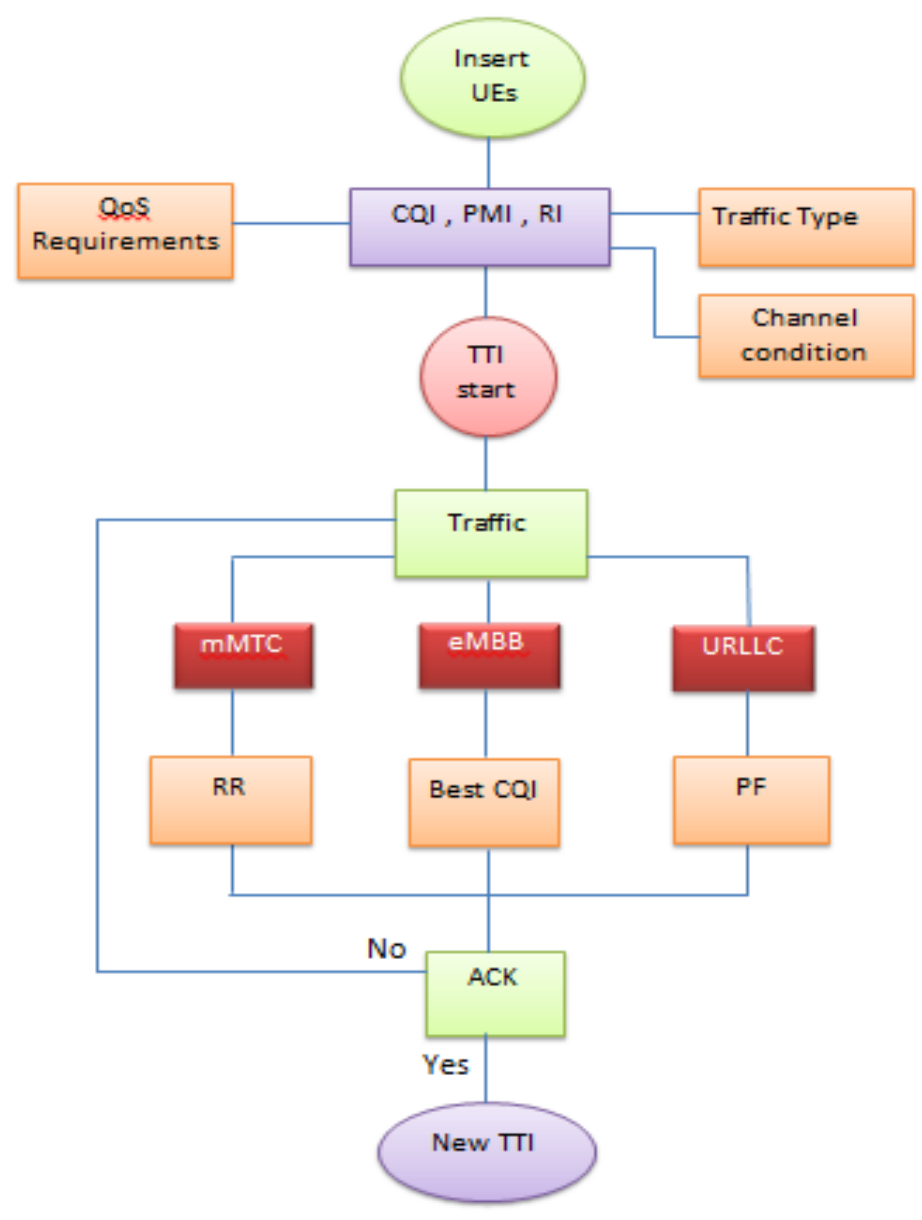

Fig 8. Flow chart of the proposed scheduling.

\subsection{Anticipated Results}

The proposed dynamic scheme is based on the traffic slicing technique to schedule the traffic user's applications. The adopted proposed scheme can be simulated, be implemented, and tested 
efficiently. It can be real-time to evaluate the $5 \mathrm{G}$ system performance at uplink utilizing network slicing.

\section{Conclusion}

In this paper, comparative resource scheduling algorithms have been evaluated for the $5 \mathrm{G}$ system. The algorithms for resource allocation has been discussed towards the $5 \mathrm{G}$ era. The paper has compared many resource scheduling algorithms to justify the optimum scheme. Then, it was concluded the best resource allocation algorithms into the new 5G networks based on math models and typical examples.

\section{References}

[1] Alliance NG. 5G white paper. Next generation mobile networks, white paper. 2015 Feb 17;1.

[2] Ferrus R, Sallent O, Perez-Romero J, Agusti R. On 5G radio access network slicing: Radio interface protocol features and configuration. IEEE Communications Magazine. 2018 Jan 10;56(5):184-92.

[3] Li X, Samaka M, Chan HA, Bhamare D, Gupta L, Guo C, Jain R. Network slicing for 5G: Challenges and opportunities. IEEE Internet Computing. 2017 Sep 18;21(5):20-7.

[4] Sayadi B, Gramaglia M, Friderikos V, Von Hugo D, Arnold P, Alberi-Morel ML, Puente MA, Sciancalepore V, Digon I, Crippa MR. SDN for 5G Mobile Networks: NORMA perspective. InInternational Conference on Cognitive Radio Oriented Wireless Networks 2016 May 30 (pp. 741753). Springer, Cham.

[5] Yousaf FZ, Bredel M, Schaller S, Schneider F. NFV and SDN-Key technology enablers for 5G networks. IEEE Journal on Selected Areas in Communications. 2017 Oct 6;35(11):2468-78.

[6] Al Janaby AO. 5G Downlink Throughput Enhancement by Beams Consolidating at Vacant Traffic. Journal of Communications Software and Systems. 2019 Dec 3;15(4):311-6.

[7] Habaebi MH, Chebil J, Al-Sakkaf AG, Dahawi TH. Comparison between scheduling techniques in long term evolution. IIUM Engineering Journal. 2013 Mar 4;14(1).

[8] Othman A, Othman SY, Al-Omary A, Al-Rizzo H. Comparative performance of subcarrier schedulers in uplink LTE-A under high users' mobility. International Journal of Computing and Digital Systems. 2015;4(04).

[9] Kawser MT, Farid HM, Hasin AR, Sadik AM, Razu IK. Performance comparison between round robin and proportional fair scheduling methods for lte. International Journal of Information and Electronics Engineering. 2012 Sep;2(5):678-81.

[10] Kumar N, Ahmad A, Awasthi S, Sharma K. Parameter aware utility proportional fairness scheduling technique in a communication network. International Journal of Innovative Computing and Applications. 2021;12(2-3):98-107.

[11] Davyt E, Muzante A, Rizzo M. A5iGnator: Framework para la Implementación de Algoritmos de Asignación de Recursos en 5G.

[12] Esswie AA, Pedersen KI. Multi-user preemptive scheduling for critical low latency communications in 5G networks. In2018 IEEE Symposium on Computers and Communications (ISCC) 2018 Jun 25 (pp. 00136-00141). IEEE.

[13] Gomez K, Goratti L, Granelli F, Rasheed T. A comparative study of scheduling disciplines in 5G systems for emergency communications. In1st International Conference on $5 \mathrm{G}$ for Ubiquitous Connectivity 2014 Nov 26 (pp. 40-45). IEEE. 
[14] Kanagasabai A. On Design and Analysis of Channel Aware LTE Uplink and Downlink Scheduling Algorithms (Doctoral dissertation, Université d'Ottawa/University of Ottawa).

[15] Dikamba T. Downlink scheduling in 3GPP long term evolution (LTE).

[16] Cuesta CF. Resource Allocation and Time-Frequency Scheduling in LTE. InSeminar Mobile Communication, TUWEIN 2007 (Vol. 12). 